\title{
Stability of PEEC Models with Respect to Partial Element Accuracy
}

\author{
Jonas Ekman ${ }^{*}$, Giulio Antonini ${ }^{\dagger}$, Antonio Orlandi ${ }^{\dagger}$, and Albert E. Ruehli ${ }^{\ddagger}$ \\ ${ }^{*}$ Luleå University of Technology, Luleå, Sweden \\ Email: Jonas.Ekman@csee.ltu.se \\ †University of L'Aquila, L'Aquila, Italy \\ Email: \{Antonini,Orlandi\}@ing.univaq.it \\ ‡IBM T. J. Watson Research Center, New York, NY 10598, USA
}

\begin{abstract}
In this paper, we examine the passivity and stability of quasi-static partial element equivalent circuit models. The impact of inaccuracies in the computed partial element values is considered as a possible source of time domain instabilities. Our analysis shows how existing partial element calculation routines, analytical and numerical, and the use of poor mesh generators can introduce large errors in partial element values. We also show how this affects the passivity and stability of the PEEC model. Theoretical constraints for passivity are derived which depend on accuracy of partial element values. The conditions are verified by performing practical PEEC model analysis.
\end{abstract}

\section{INTRODUCTION}

With the increased performance of todays electronic systems, electromagnetic (EM) modeling is of increasing importance for EMC researchers and product developers. These problems can be solved efficiently by choosing an appropriate modeling technique. Solving combined electromagnetic and circuit analysis problems is required for printed circuit board (PCB), subsystem-PCB modeling, and electrical interconnect and package (EIP) problems. Two dimensional (2D) transmission line analysis is used when applicable. However, where 2D modeling is inadequate, three dimensional (3D) modeling techniques must be used. In the 2003 International Technology Roadmap for Semiconductors (TTRS) [1], the use of highfrequency 3D EM modeling is designated as an emerging area. This roadmap states that the need for high-frequency modeling and package electrical modeling will be of increasing importance over the next decade. Some of the reasons cited are the multi-GHz signal bandwidths at all levels of integration and packaging, mixed-signal functionality, and larger wiring densities in complex 3D environments [2]. The partial element equivalent circuit (PEEC) method [3]-[5] is a $3 D$ full wave modeling method for these type of problems. Unlike the method of moments (MoM), the PEEC is a full spectrum method valid from $d c$ to the maximum frequency determined by the meshing.

In the PEEC method, the integral equation is interpreted as Kirchoff's voltage law applied to a basic PEEC cell which results in a complete circuit solution for 3D geometries. The benefit from the equivalent circuit formulation is that additional SPICE type circuit elements can easily be included. Also, the models and the analysis apply to both the time and the frequency domain. Other advantages of the PEEC circuit formulation are the direct applicability of model order and/or model complexity reduction. With a general purpose SPICE like solver, different analysis such as full-wave or static, LR or $\mathrm{RC}$, and transmission model analysis can be performed. The PEEC method has recently been extended to include nonorthogonal geometries [6]. This model extension, which is consistent with the classical orthogonal formulation, enables better modeling of non-orthogonal geometries by avoiding the typical staircase approximations previously used and thereby improves modeling accuracy. The MNA formulation [7] solves simultaneously for the volume cell currents and the node potentials in the discretized structure. This approach is used in most SPICE type circuit solvers. It has several advantages over an MoM analysis and is preferable to an admittance formulation [8] since any type of circuit element can be included in a straightforward way with appropriate matrix stamps.

In this paper, we investigate the time domain stability analysis of PEEC based electromagnetic models from a circuit perspective. Here we concentrate on the the impact of the accuracy in the partial elements on the stability. This type of stability analysis has been motivated by several issues.

- The recently introduced nonorthogonal PEEC formulation [6] requires the use of numerical space integration rather than analytical formulas as they are used for most partial element evaluation.

- Stability is an important and difficult issue for full-wave PEEC solvers.

- PEEC modeling is used for a wide variety of geometries. Some of these systems result in dense PEEC model submatrices with a wide range of coupling terms which are prone to numerical errors.

- Recently, research on the sparsification of partial inductance matrices has been attempted [9] by windowingtechniques [10] and wire-duplication [11]. They investigated the stability impact of the partial inductance matrix elements.

- The modeling of more complex systems requires projection meshing (see Sec. IV-B) to assure correctness of the partial element values [12].

This paper is organized in the following manner. In the 
next section, we consider the PEEC model and model stability analysis and the different sources contributing to erroneous partial elements are detailed in Section IV. Section V detail the passivity and stability of PEEC models with respect to partial coefficients of potential. A symmetric PEEC one-cell is studied for the electromagnetic quasi-static case resulting in conditions for the lumped capacitance and partial coefficient of potential values for which the structure is stable or unstable. Practical examples are given for stable and unstable quasistatic PEEC models with respect to the derived theoretical conditions. Finally, conclusions are drawn.

\section{PEEC MOdel DeRivation}

The single dielectric form of the PEEC method is derived from the mixed potential integral equation (MPIE) written as

$$
\vec{E}^{i}=\frac{\vec{J}(\vec{r}, t)}{\sigma}+\frac{\partial \vec{A}(\vec{r}, t)}{\partial t}+\nabla \phi(\vec{r}, t)
$$

where $\vec{E}^{i}$ is an incident electric field, $\vec{J}$ is a current density, $\vec{A}$ is the vector magnetic potential, and $\phi$ is the scalar electric potential at observation point $\vec{r}$. By using the definitions of the EM potentials, the current- and charge- densities are discretized by defining pulse basis functions for the conductors and dielectric materials. Pulse functions are also used for the weighting functions resulting in a Galerkin solution and the interpretation of constant current- and charge- densities over the discretized cells. By defining a suitable inner product with a weighted volume integral over the cells, the MPIE in (1) can be interpreted as KVL over a PEEC cell consisting of:

- partial self inductances between the nodes and partial mutual inductances representing the magnetic field coupling in the equivalent circuit. The partial inductance is defined as

$$
L p_{\alpha \beta}=\frac{\mu}{4 \pi} \frac{1}{a_{\alpha} a_{\beta}} \int_{v_{\alpha}} \int_{v_{\beta}} \frac{1}{\left|\vec{r}_{\alpha}-\vec{r}_{\beta}\right|} d v_{\alpha} d v_{\beta}
$$

- coefficients of potential to each node and mutual coefficients of potentials between the nodes representing the electric field coupling. The coefficients of potentials are defined as

$$
p_{i j}=\frac{1}{S_{i} S_{j}} \frac{1}{4 \pi \epsilon_{0}} \int_{S_{i}} \int_{S_{j}} \frac{1}{\left|\vec{r}_{i}-\vec{r}_{j}\right|} d S_{j} d S_{i}
$$

- a resistive term between the nodes, defined as

$$
R_{\gamma}=\frac{l_{\gamma}}{a_{\gamma} \sigma_{\gamma}}
$$

Common for (2) and (4) is that $a$ represents the cross section of the volume cell normal to the current direction $\gamma$ and $l$ is the length in the current direction. Further, $v$ represents the current volume cells and $S$ the charge surface cells. For a detailed derivation of the method which includes the nonorthogonal formulation, see [6].

\section{INTRODUCTION TO PEEC MODEL STABILITY}

ANALYSIS

The time domain stability problem for PEEC models, and other integral equation formulated EM analysis methods is well known, e.g. [13]. This well known problem, which is often termed 'late time instability' results in an oscillation of an exponentially increasing amplitude which starts at some point in time while totally masking the real solution. Reasons for the instabilities are:

1) the meshing of the geometry which is also related to the delay discretization for full wave models, e.g. [14];

2) the numerical time domain integration technique [15] which is important for both quasi-static and full-wave PEEC models.

Both issues have been published and solutions were proposed to improve the stability of PEEC models based on these analyzes. Here, we introduce a third issue which impacts on PEEC model stability, which is the accuracy of the partial element values. It will be shown in the following sections that the factors contributing to inaccuracies in partial element calculations are:

- the wrong application of calculation routines applicable only for specific cases;

- the use of large-aspect-ratio PEEC cells for which certain calculation routines perform poorly;

- the use of inadequate numerical integration for general PEEC and nonorthogonal PEEC models;

- the inappropriate discretization of objects without adapted meshing routines.

The consequences of erroneous calculated partial elements in the final PEEC model solution are hard to predict. However, there is a large possibility that the errors will be emphasized when creating the PEEC circuit equations where numerical errors can be introduced in the required matrix operations. The result can be an unstable PEEC model or a stable PEEC model providing incorrect information. This paper focuses on the errors introduced in partial element calculation and the numerical errors introduced by the matrix operations.

\section{SoURCES OF ERRoneous Partial ElEMENT VAlues}

\section{A. Calculation Routines}

This section addresses the importance of using the right routines when calculating the partial elements. Examples on unstable and unsuitable routines are given for both the analytical and numerical routines.

I) Analytical formulas: The use of analytical formulas for partial element calculation in PEEC modeling is desirable due to fast and simple usage. For orthogonal PEECs, the analytical formulas are unconditionally used. However, the formulas have to be used with care. One of the benefits with PEEC modeling is the possibility to use large-aspect-ratio cells in the discretization. This requires the use of correct routines so that numerical instabilities, as illustrated in Fig. 1, can be avoided. Fig. 1 gives an example of how an analytical formula, used to calculate the partial self inductance for a PEEC model volume 
cell, can produce totally wrong inductance values, including negative results for the partial self inductance.

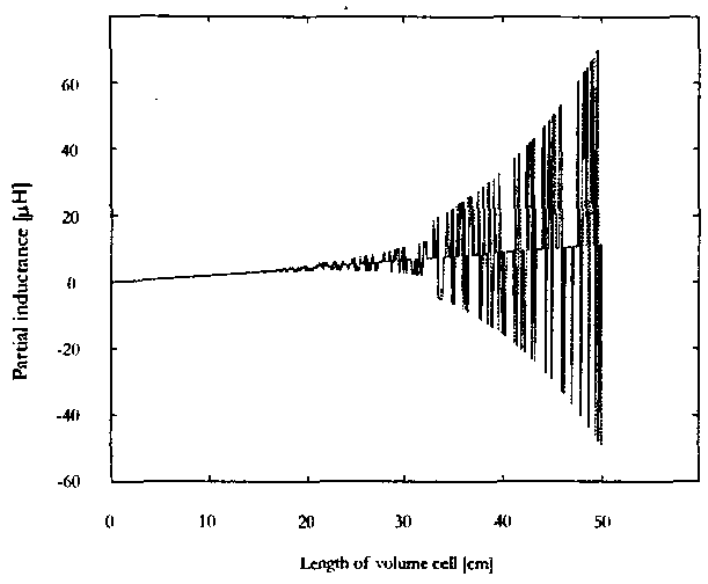

Fig. 1. Numerical unstable partial self inductance calculation routine, constant cross-section with increasing length.

Previously published guidelines [16] have proved to be valuable when choosing analytical formulas for the partial coefficient evaluation.

2) Numerical integration: Numerical integration for the evaluation of the basic partial element expressions is used for nonorthogonal structures or when analytical formulas are difficult to find. The common use of large-aspect-ratio cells and/or large separation distances put difficult constraints on the numerical integration routines. Hence, an adaptive strategy must be used to accommodate wide variations in the coefficients. Further, the wide range of approximations for the basic partial element expressions, surface- and contourformulations, can help from an efficiency point of view but can introduce problems when not used correctly.

Fig. 2 shows the calculated partial self inductance for an orthogonal cell with $0.01 \times 0.01 \mathrm{~cm}$ cross section as a function of length (x-axis) using the proposed approach in [16] using analytical functions in comparison to a $3 \mathrm{D}$ (volume) formulation using 5 th or 8th order of Gauss-Legendre (G-L) numerical integration. It is clear that even the 8 th order of Gauss-Legendre numerical integration gives poor results for conductor aspect ratios of $1: 2$ since the relative error in the order of $1.5 \%$ is much too large for dense problems. Also, a very careful implementation is required to avoid large compute time when using the 8th order or higher G-L numerical integration techniques.

The previous results displayed the self inductance for a volume cell with finite thickness. Since zero-thickness structures are sometimes sufficient for some problems. For this reason, a test was performed for a 'zero-thickness' cell. The results shown in Fig. 3 compare the same scheme used in the previous example, using conditioning of analytical formulas, with the contour formulation [17] using 5th or 8th order of Gauss-Legendre numerical integration. The results show the same trend as for the finite thickness case in the previous example. We can define a limit for conductor length to width ratio of max. 2:1 using 5th order G-L numerical integration. The contour formulation is much faster than the volume formulation previously. Hence, they show an advantage for the close form solutions for PEEC solvers.

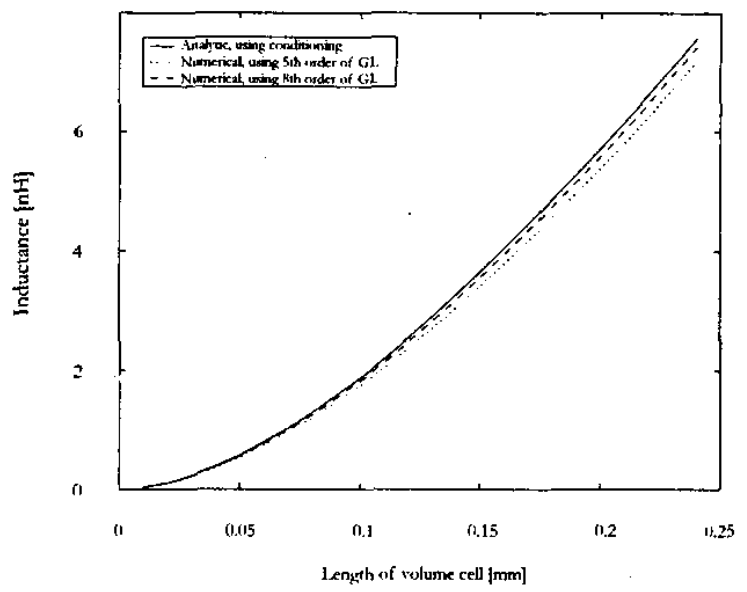

Fig. 2. Partial self inductance calculation using analytical (exact) or numerical (volume) techniques.

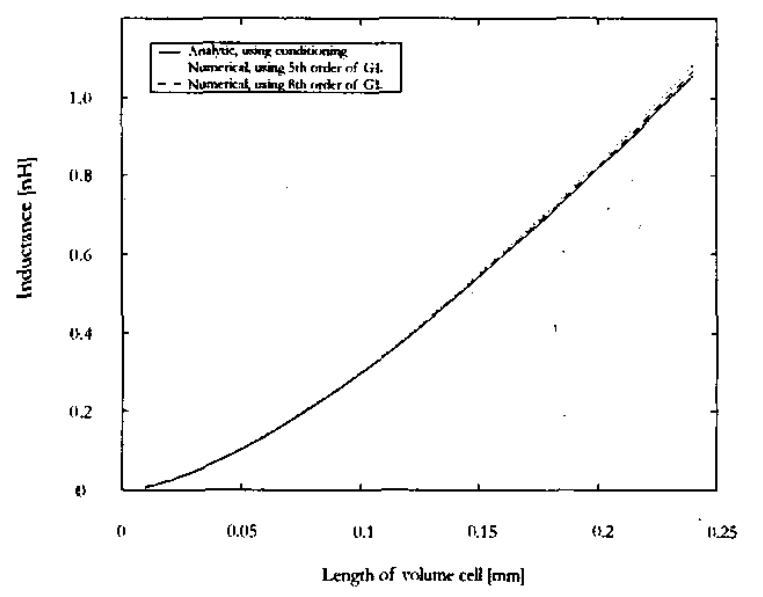

Fig. 3. Partial self inductance calculation using analytical (exact) or numerical (contour) techniques.

From the tests results presented in Figs. 2 and 3, important conclusions can be drawn:

- using the volume formulation with low order of GaussLegendre numerical integration underestimates the partial self inductance of a rectangular conductor;

- second, the contour formulation overestimates the partial self inductance of a zero-thickness conductor.

The consequences of over- or under-estimating the partial self inductance can be important (a source of instabilities) when mixing analytical and numerical based calculation routines for large problems of mixed orthogonal and nonorthogonal objects. Both over- and under-estimating partial self elements 
influences the partial inductance matrix, $\mathbf{L}_{p}$, which must be positive definite. For example, by underestimating self terms can result in mutual terms that are larger and thus creating an indefinite $\mathbf{L}_{p}$-matrix.

This section has illustrated the potential error in partial inductance calculations using different available formulations. The partial coefficient of potential (capacitive) calculation is very similar and similar results are expected when performing the same tests.

For orthogonal PEEC models, the partial elements are best calculated using schemes of the type presented in [16]. For nonorthogonal elements, when numerical integration is used, the required integration order needs to be examined and schemes developed to assure accurate partial elements and modeling results.

\section{B. Discretization of Geometry}

One of the objectives of the meshing in the PEEC approach is the careful allocation of the unknowns or subdivisions. A technique which is called projection meshing is used to obtain convergent results without an excessive number of cells. In this approach, cells for neighboring conductors are lined up. It was shown in [12] that capacitive couplings could be completely wrong if projection meshing was not used for close conductors. It is evident that the proper meshing for multiple conductor geometries is quite a challenge.

In this section, we give an example similar to the one in [12], to display the importance of projection meshing for PEEC models. Consider the three conductor geometry in Fig. 4. The lower plate, No.1, is $10 \times 5 \mathrm{~cm}$; the upper left plate, No.2, is $3 \times 5 \mathrm{~cm}$; and the upper right plate, No.3, is $5 \times 5$ $\mathrm{cm}$. The gap separation between the upper plates No.2 and No.3 is $1 \mathrm{~cm}$ and the inter-plane separation is $0.2 \mathrm{~cm}$. The mutual coupling between the plates is given by the short circuit capacitance matrix $\mathbf{C}_{S}$ given in Section V-A. To find $\mathbf{C}_{S}$, we analytically compute the partial coefficients of potentials [4]. Importantly, here we assume that the plates are not subdivided into smaller cells to use the smallest number of unknowns, which is three. Then, the matrix of potential coefficients is given by

$\mathbf{P}=\left[\begin{array}{lll}0.367179683 & 0.355179322 & 0.346424918 \\ 0.355179322 & 0.679185447 & 0.185787332 \\ 0.346424918 & 0.185787332 & 0.534448842\end{array}\right]\left[p F^{-1}\right]$

which results in a short circuit capacitance matrix according to

$$
\mathbf{C}_{S}=\left[\begin{array}{rrr}
18.88094007 & -7.21178058 & -9.73146582 \\
-7.21178058 & 4.38168922 & 3.15143006 \\
-9.73146582 & 3.15143006 & 7.08342159
\end{array}\right][p F]
$$

This result is clearly in error since this matrix is supposed to be a positive definite Minkowski M-matrix with all its off diagonals being negative. If we subdivide the lower plate, No.1, into four parts which projects the shape of the two upper plates, then the capacitive couplings are calculated much more accurately and the resulting short circuit capacitance matrix is

$$
\mathbf{C}_{S}=\left[\begin{array}{rrr}
21.3179316 & -7.6205393 & -12.0063976 \\
-7.6205393 & 8.1683522 & -0.1459876 \\
-12.0063976 & -0.1459876 & 12.8934069
\end{array}\right][p F]
$$

This simple example shows the possible errors introduced in the partial element values by not using a projection meshing type approach where neighboring cells are matched in the limit as the distance between the conductors goes to zero.

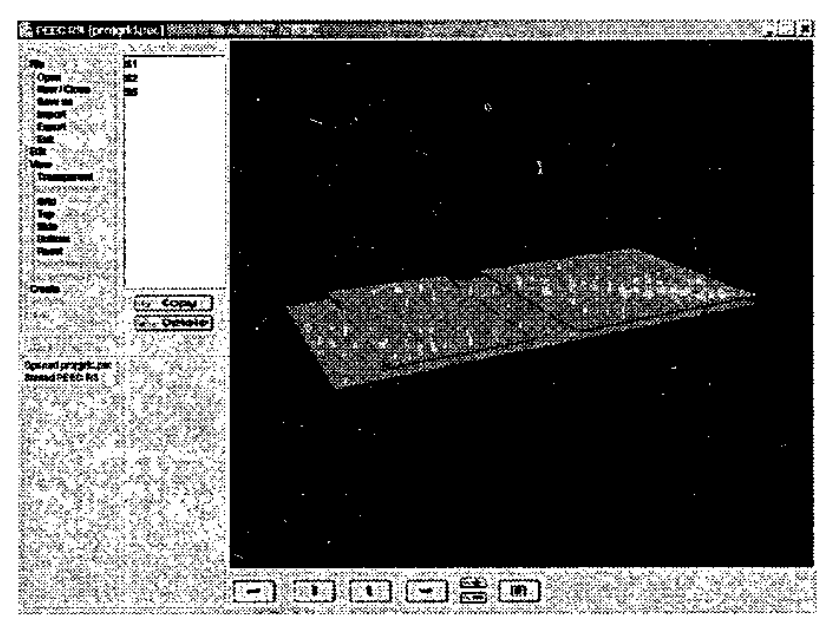

Fig. 4. Three conductor geometry.

In the next section, the passivity and stability of simple PEEC models are investigated with respect to accuracy of the calculated partial coefficient of potential values. It will be evident that the proper meshing does have an impact on the stability issue.

\section{STABILITY With RESPECT TO Potential COEFFICIENTS}

\section{A. Theoretical considerations}

The electric field or the capacitive effects in PEEC are modeled using the theory of partial coefficients of potential, $p_{i j}$, calculated using the basic expression in (3). In the PEEC method, a coefficient of potential matrix, $\mathbf{P}$, is constructed with elements of the type $p_{i j}$, which is more dense than the partial element matrix for rectangular geometries. For PEEC, the $\mathbf{P}$ matrix is dense, symmetric, and positive definite [4]. The energy for the potential coefficient can be written as a quadratic form, or

$$
W_{e}=\frac{1}{2} \mathbf{Q}^{T} \mathbf{P Q}
$$

where $\mathbf{Q}$ is the vector of total cell surface charge. Note all elements in $\mathbf{P}$ are positive as is evident from (3). For a quasistatic PEEC model, lumped capacitances can be used from the inverse of the $\mathbf{P}$ matrix or

$$
\mathbf{C}_{S}=\mathbf{P}^{-1}
$$


where the $\mathbf{C}_{S}$ is a short circuit capacitance M-matrix with positive diagonal and negative off diagonal elements. Hence, it is evident that any positive off diagonal element in the $\mathbf{C}_{S^{-}}$ matrix indicates an error in the analysis which may be caused by meshing or a combination of strong and weak couplings, as well as numerical errors in the coefficients. The circuit twoterminal capacitances are obtained by manipulating the $\mathbf{C}_{S}$ matrix according to the following:

- the diagonal terms are obtained by summing each row in the $\mathbf{C}_{s}$-matrix, $c_{i i}=\sum_{\forall j} c_{s_{i j}}$;

- the off diagonal terms are the negative value for the corresponding off diagonal element in the $\mathbf{C}_{s}$-matrix, ie. $c_{i j}=-c_{s_{i j}}$.

Thus, it is evident that all two terminal circuit capacitances should be positive.

Here, the stability of a quasi-static PEEC model with respect to circuit capacitances for a simple one-cell PEEC structure is considered, Fig. 5. It is well known from circuit theory that lumped circuits without independent as well as dependent sources and with positive circuit elements are passive. We use the single cell model to illustrate the impact of inaccurate potential coefficients on stability. To monitor the stability and passivity of the circuit, we study the input impedance at the point marked $Z_{I N}$ in. Fig.5. It is evident that both nodes in the small circuit are observable from this point. Stability and passivity are given by the condition that all eigenvalues are in the left hand plane, or $\operatorname{Re}\left(Z_{I N}\right) \geq 0$ for all frequencies since a negative input impedance indicates a non-passive circuit which generates energy. The input impedance for the one-cell model

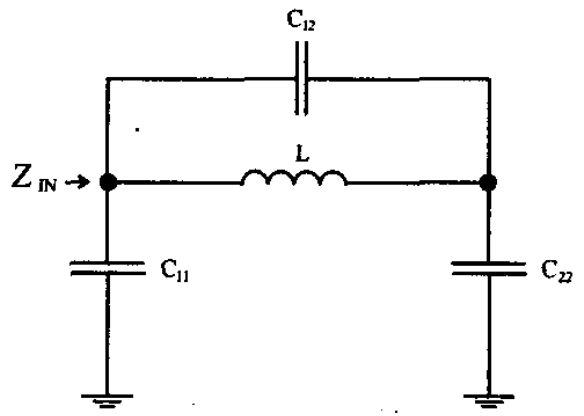

Fig. 5. Simple PEEC circuit used for quasi-static EM problems.

is given by

$$
Z_{I N}=\frac{s^{2} L C_{22}+s^{2} L C_{12}+1}{s\left(s^{2} L\left(C_{11} C_{22}+C_{12}\left(C_{11}+C_{22}\right)\right)+C_{11}+C_{22}\right)},
$$

with passivity condition

$$
C_{12}>-\frac{C_{11} C_{22}}{C_{11}+C_{22}}
$$

If the above condition is not fulfilled, the one-cell system can have positive eigenvalues corresponding to the poles of the transfer function $Z_{I N}$, causing the system to be unstable. The next section explores the practical implications of the passivity criterion developed above by modeling the one-cell in PSpice [18] for a few different cases.

While our analysis is restricted to quasi-static PEEC models, we can argue that the conditions are necessary for fullwave (Lp,R,P, $\tau$ )PEEC models where $\tau$ indicates that delays or retardation is used. Essentially, retardation is negligible for sufficiently low frequencies or for sufficiently small geometries. For these cases the quasi-static conditions must be fulfilled.

\section{B. Numerical Experiments}

First a symmetric one-cell geometry is considered. The length is $5 \mathrm{~cm}$, the width is $2 \mathrm{~cm}$, and the thickness is very small, $1 \mu \mathrm{m}$, and is assumed to be zero thickness in the calculations. The PEEC model matrices are as follows:

$$
\begin{gathered}
\mathbf{P}=\left[\begin{array}{ll}
1.19146 & 0.41638 \\
0.41638 & 1.19146
\end{array}\right] \quad\left[p F^{-1}\right] \\
\mathbf{C}=\left[\begin{array}{ll}
0.622 & 0.334 \\
0.334 & 0.622
\end{array}\right] \quad[p F]
\end{gathered}
$$

and $\mathrm{L}_{p}=22.36 \mathrm{nH}$. The one-cell is excited using a unit current source at the node indicated with $Z_{I N}$. Figures 6 and 7 show the responses for different mutual capacitance, $C_{12}$, values. These results are consistent with the theoretical constraints developed earlier.

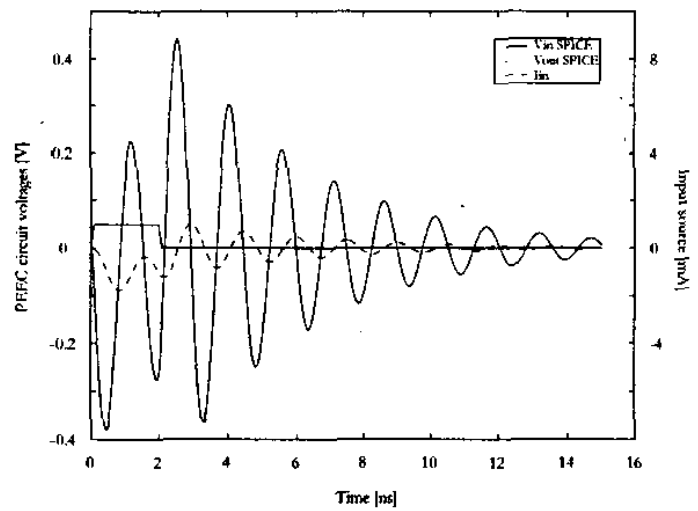

Fig. 6. Stable response for symmetric one-cell with $C_{12}=0.334 \mathrm{pF}$.

We next consider the one-cell with an asymmetric discretization where the coefficients of potentials are calculated from two different sized cells. As an example, we choose 4.75 $\mathrm{cm}$ and $0.25 \mathrm{~cm}$ for the two lengths resulting in the following PEEC model matrices:

$$
\begin{gathered}
\mathbf{P}=\left[\begin{array}{ll}
0.82921 & 0.50647 \\
0.50647 & 2.97818
\end{array}\right] \quad\left[p F^{-1}\right] \\
\mathbf{C}=\left[\begin{array}{ll}
0.112 & 0.023 \\
0.023 & 0.015
\end{array}\right] \quad[p F]
\end{gathered}
$$

and $\mathrm{L}_{p}=22.36 \mathrm{nH}$ as before. The one-cell is excited using the same current source as before resulting in the responses in Figs. 8 and 9. 


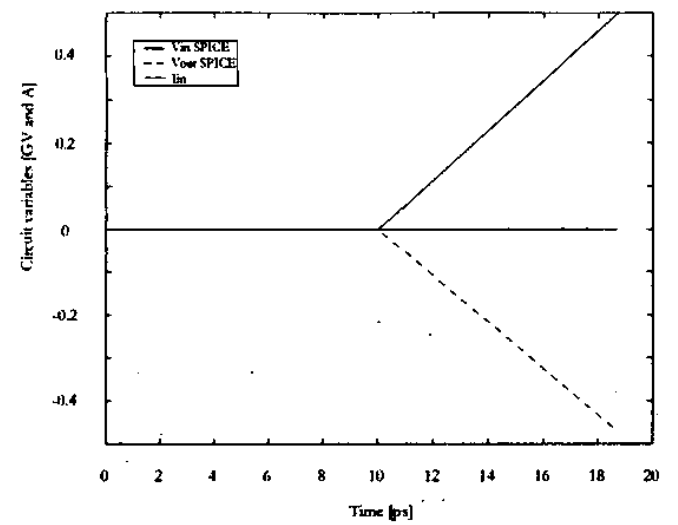

Fig. 7: Unstable response for symmetric one-cell with $C_{12}=-0.32 \mathrm{pF}$, violating passivity condition in (11).

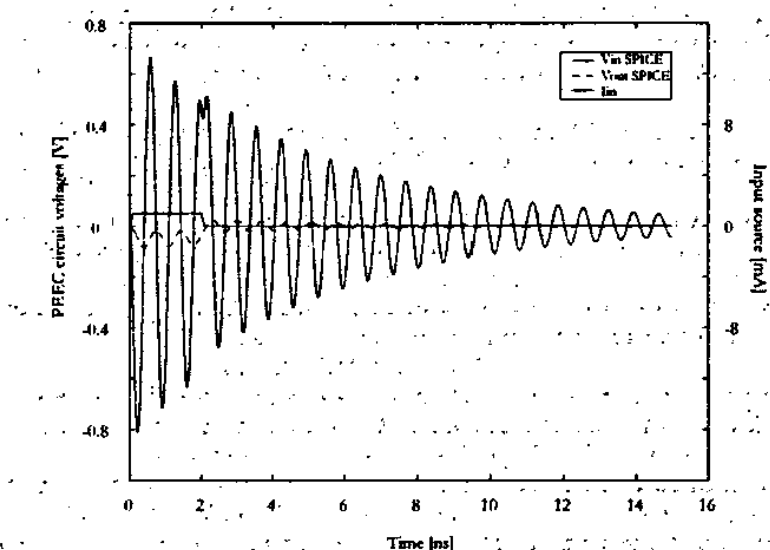

Fig: 8. Stable response for asymmetric one-cell with $C_{12}=0.023 \mathrm{pF}$

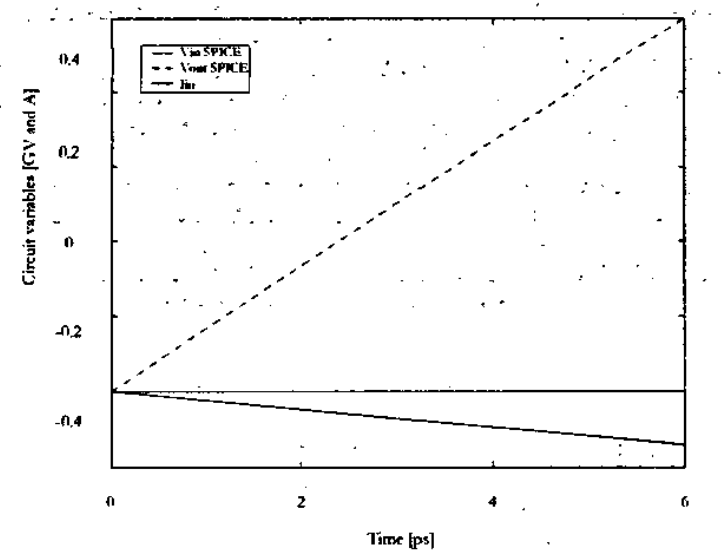

Fig. 9. Unstable response for asymmetric one-cell with $C_{12}=-0.014 \mathrm{pF}$, violating passivity condition in (11).

The results are in accordance with the theoretical constraints developed earlier. Importantly, we conclude that the asymmetric discretization put a stronger constraint on the accuracy in the mutual capacitance term, $C_{12}$. This corresponds to realistic models since the cell size is widely varying for practical problems.

\section{CONCLUSIONS}

This paper extends the stability analysis of quasi-static PEEC models to include accuracy of calculated partial elements. The specific reasons are erroneous partial elements, analytical and numerical calculation routines and poor meshing of geometries. Further, the impact of inaccurate partial elements are shown to be a source of instabilities. Poor meshing may result in negative capacitance values as possible source of instabilities. The paper gives references to suitable partial element calculation routines and a meshing strategy to overcome sources of instabilities.

\section{REFERENCES}

[1] (2003) International technology roadmap for semiconductors 2003 edition, ITRS2003. - [Online]. : Available: http://public.itrs.net/Files/2003ITRS/Home2003.htm

[2] V. Okhmatovski, A. Cangellaris, and J. Morsey, "Accuracy-related issues in electronic modeling of high-speed interconnects," in Proc. of the IEEE International Symposium on EMC. Boston, MA, USA; 2003, pp. 338346.

[3] A. E. Ruehli, "Inductance calculations in a complex integrated circuit environment," IBM Journal of Research and Development, vol. 16, no. 5 , pp. $470-481$, Sept. 1972.

[4] A. E. Ruehli and P. A. Brennan,. "Efficient capacitance calculations for three-dimensional multiconductor systems," IEEE Trans. Microwave Theory Tech., vol. 21, no. 2, pp. 76-82, Feb. 1973

[5] A. E. Ruehli, "Equivalent circuit models for three dimensional multiconductor systems," IEEE Trans. Microwave Theory Tech., vol. 22, no. 3, pp. 216-221, Mar. 1974.

[6] A. E. Ruehli, et al., "Nonorthogonal PEEC formulation for time- and frequency-domain EM and circuit modeling," IEEE Trans. Electromagn. Compat., vol. 45, no. 2, pp. 167-176, 2003.

[7] C. Ho, A. Ruehli; and P. Brennan, "The modified nodal approach to network analysis," IEEE Trans. Cincuits Syst., pp. 504-509, June 1975.

[8] T. A.- Jerse, "A hybrid technique for efficiently estimating common mode currents in transmission-line structures," Ph.D. dissertation, The University of Kentucky, 1994.

[9] B. Krauter and L. T. Pileggi, "Generating sparse partial inductance matrices with guaranteed stability," in Proc. of Int. Conf. on Computer Aided Design, San Jose, CA, USA, 1995, pp. 45-52.

[10] M. Beattie and L. Pileggi. "Efficient inductance extraction via windowing," in Proc. of Design, Automation and Test in Europe, Munich, Germany. 2001, pp. 430-436.

[11] G. Zhong, C.-K. Koh, and K. Roy, "On-chip interconnect modeling by wire duplication," in Proc. of Int. Conf. on Computer Aided Design, San Jose, CA, USA, 2002, pp. 341-346.

[12] A. E. Ruehli, P. A. Brennan, and H. W. Young, "Recent progress in capacitance computation methods," in Proc. of the IEEE Int. Symposium on Circuits and Systems, Phoenix, AZ, USA, 1975, pp. 135-138.

[13] B. Rynne, "Comments on a stable procedure in calculating the transien scattering by conducting surfaces of arbitary shape," IEEE Trans. Antennas Propagat, vol. 41, no. 4, pp. 517-520, Apr. 1993.

[14] A. Cangellaris, W. Pinello, and A. E. Ruehli, "Stabilization of time domain solutions of EFIE based on partial element equivalent circuit models," in Proc. of the IEEE Antennas and Propagation Society Int. Symposium, Montréal, Canada, 1997. pp. 966-969.

[15] A. E. Ruehli, et al., "Stable time domain solutions for EMC problems using PEEC circuit models." in Proc. of the IEEE Int. Symposium on EMC, Chicago, IL, USA, 1994, pp. 371-376.

[16] P. K. Wolff and A. E. Ruehli. "Inductance computations for complex three dimensional geometries," in Proc. of the IEEE Int. Symposium on Circuits and Systems, New York. NY, USA, 1981, pp. 16-19.

[17] G. Antonini. A. Orlandi. and A. E. Ruehli, "Analytical integration of quasi-static potential intergrals on nonorghogonal coplanar quadrilaterals for the PEEC method," IEEE Trans. Electromagn. Compat., vol. 44 no. 2, pp. 399-403, May 2002.

[18] (2003) PSpice A/D datasheet. [Online]. Available: http:/www.orcad.com/pdf/PSpiceAD.DS.pdf 